\title{
Prematuros tardíos: estudio clínico retrospectivo de sus causas y consecuencias
}

\author{
Juan F. Stecher M. ${ }^{1,2}$, Milenko Pavlovic B. ${ }^{1}$, Cristian Contreras V. ${ }^{1}$, Jorge Carvajal C. ${ }^{1}$ \\ ${ }_{2}^{1}$ División de Ginecología y Obstetricia, Escuela de Medicina, Pontificia Universidad Católica de Chile. Santiago, Chile. \\ ${ }^{2}$ Facultad de Medicina, Universidad Católica de la Santísima Concepción. Concepción, Chile.
}

\section{RESUMEN}

Antecedentes: Los niños nacidos entre las 34 0/7 y 36 6/7 semanas se denominan prematuros tardíos (PT), constituyen 5-7\% de los nacidos y poseen mayor morbimortalidad que los niños de término. Objetivo: Analizar las causas de partos PT en nuestra institución. Comparar morbilidad neonatal de nacidos PT y de término. Métodos: Estudio de cohorte retrospectivo. Se revisan registros de nacimientos entre enero de 2009 y diciembre de 2012 identificando los nacidos vivos entre las 34 0/7 y 36 6/7 y entre las 39 0/6 y 40 $6 / 7$ semanas. Se identifica grupo clínico de parto prematuro al que pertenecen. Se compara frecuencia de resultados perinatales de nacidos PT y de término completo. Resultados: En el período estudiado nacieron 8.890 niños vivos. Tasa de partos PT fue 7,49\% $(n=666)$. El $64,11 \%$ fue por causa idiopática o asociado a rotura de membranas y $35,89 \%$ por indicación médica. Dentro del grupo de indicaciones médicas un $19,25 \%$ de interrupciones no están basadas en evidencia y ninguna puramente electiva. Los PT, comparados con niños de término, tienen más riesgo de hospitalización y morbilidad neonatal; este riesgo es mayor a menor edad gestacional. Conclusiones: En nuestro hospital 1 de cada 5 PT pudo haber nacido a una edad gestacional mayor, lo que podría haber evitado los riesgos perinatales asociados. Se sugiere que las instituciones de salud analicen las causas de interrupciones del embarazo en el grupo de PT, lo que podría ser un indicador de calidad obstétrica.

\section{PALABRAS CLAVE: Prematuros tardíos, morbilidad, prematurez iatrogénica, epidemiología}

\section{SUMMARY}

Background: Children born between 34 0/7 and 36 6/7 weeks of gestation are called late-preterm infants. Represent 5 to $7 \%$ of live births and they are at higher risk of morbidity and mortality than term infants. Objective: To analyze causes of late preterm births in our institution. To compare neonatal morbidity in late-preterm and term infants. Methods: A retrospective cohort study. Birth records between January 2009 and December 2012 were reviewed identifying live births between 34 0/7 and 36 6/7 and between 39 0/6 and 40 6/7 weeks of gestation. Clinical group of preterm labor to which they belong was identified. Frequency of perinatal outcomes of late-preterm and term infants were compared. Results: In the period under study there were 8890 live birth. The late-preterm birth rate was $7.49 \%(n=666) ; 64.11 \%$ of them was idiopathic in cause or associated with rupture of membranes and $35.89 \%$ was for medical indication. In the group of medical indications $19.25 \%$ of the interruptions were not based on evidence and none were purely elective. Late-preterm infants have higher risk of hospitalization and neonatal morbidity than term infants; this risk is higher at earlier gestational ages. Conclusions: In our hospital 1 in 5 late-preterm birth could have been born at higher gestational age, preventing perinatal risks. It is suggested that health institutions analyze causes of interruption of pregnancy in the late-preterm group, which could constitute an indicator of obstetric quality.

KEY WORDS: Late preterm, morbidity, iatrogenic premature birth, epidemiology 


\section{INTRODUCCIÓN}

Los partos prematuros son aquellos que ocurren antes de las 37 semanas de edad gestacional; se conoce como prematuros tardíos (PT), a los nacidos entre las 34 0/7 y 36 6/7 semanas desde la fecha de última menstruación. En Chile en 2009, el $7,2 \%$ de los partos fue menor a las 37 semanas, un $70 \%$ de ellos fueron partos PT (1).

La prematuridad es una importante causa de morbimortalidad perinatal y una de las principales causas de problemas neurológicos en la infancia. Los PT, si bien suelen evolucionar favorablemente, presentan mayor mortalidad y morbilidad perinatal que los niños de término (>37 semanas) $(2,3)$; tienen más altas tasas de hospitalización durante el primer mes y año de vida, con mayores costos en su atención $(4,5)$; así mismo, presentan una frecuencia más alta de alteraciones neurológicas y del aprendizaje durante la infancia (6-8).

Existen 3 grupos clínicos de parto prematuro: a) trabajo de parto prematuro (idiopáticos), b) rotura prematura pretérmino de membranas, y c) indicación médica. Este último grupo ha crecido en comparación con los otros, debido principalmente a un aumento en los embarazos denominados de alto riesgo y a una mayor comprensión de las patologías del embarazo (9-11). Se ha reportado que entre los PT por indicación médica, existe un número no despreciable de interrupciones del embarazo no justificadas medicamente, lo que ha llevado a aumentar las tasas de partos de pretérmino sin un beneficio claro para los niños o sus madres (12-14).

En Chile se publicó un estudio de cohorte que analizó la morbilidad neonatal de los PT (15), con resultados concordantes con lo reportado en la literatura internacional. Sin embargo, no existen reportes nacionales acerca del grupo clínico al cual pertenecen los PT; especialmente, no se ha reportado la incidencia de la indicación médica como causa de un PT.

El objetivo de este estudio es analizar el grupo clínico de los partos PT en nuestra institución, con un énfasis especial en analizar la causa de la indicación médica para la interrupción del embarazo. Como objetivo secundario, evaluamos la morbilidad neonatal de los PT (34 0/7 y 36 6/7 semanas) y la comparamos con la de los recién nacidos de término completo (39 0/7 - 40 6/7 semanas).

\section{PACIENTES Y MÉTODOS}

Metodología: Estudio de cohorte retrospectivo; aprobado por el comité de ética en investigación de la Facultad de Medicina de la Pontificia Universidad Católica de Chile (№ proyecto 13-015). Revisamos los registros de partos de la Maternidad del Hospital Clínico de la Universidad Católica entre enero de 2009 y diciembre de 2012 identificando a todos los nacidos vivos entre las $340 / 7$ y 36 6/7 y aquellos nacidos entre las 39 0/6 y 40 6/7 semanas. De cada uno de los niños nacidos PT, analizamos la ficha de hospitalización de la madre para identificar a que grupo clínico de parto prematuro pertenecen. En aquellos del grupo indicación médica, analizamos la causa de la interrupción y la clasificamos como: basada en la evidencia, no basada en la evidencia y puramente electiva. Examinamos la base de datos con los egresos del servicio de Neonatología, durante el mismo período de tiempo, para seleccionar a los niños nacidos entre las 34 0/7 y 36 6/7 y los nacidos entre las 39 0/7 y 40 6/7 semanas de edad gestacional que fueron hospitalizados. Analizamos la morbilidad y mortalidad de todos los recién nacidos hospitalizados y asumimos que los no hospitalizados no presentaron morbilidad. Excluimos del análisis de morbilidad y mortalidad a los niños hospitalizados por causa materna, los que presentaban malformaciones congénitas graves y aquellos con aneuploidías.

Grupos clínicos de parto prematuro: a) idiopático o trabajo de parto prematuro (primer evento detectable es aparición de contracciones uterinas regulares lo que produce el parto), b) rotura prematura de pretérmino de membranas (primer evento detectable es la rotura de membranas; secundariamente aparecen contracciones uterinas que conducen al parto), y c) indicación médica (interrupción del embarazo por indicación del médico).

Causa de la interrupción en el grupo indicación médica: a) basada en la evidencia (patología materna o fetal cumple con los criterios para interrupción del embarazo de la Sociedad de Medicina Materno Fetal y el National Institute of Child Health and Human Development (16)), b) no basada en la evidencia (la patología materna o fetal no cumple con los criterios para interrupción del embarazo a la edad gestacional en que se indicó), c) puramente electiva (no existe evidencia de patología médica o fetal que justifique la interrupción).

Morbilidad neonatal: necesidad de hospitalización en unidad de neonatología; hospitalización para observación (hospitalización sin diagnóstico específico, para observar al recién nacido); síndrome de distrés respiratorio (dificultad respiratoria progresiva de aparición precoz, caracterizado por quejido, aleteo nasal, retracción, polipnea y grados variables de compromiso de la oxigenación); enfermedad de membrana hialina (síndrome de distrés respiratorio con radiografía compatible); taquipnea transitoria neonatal (dificultad respiratoria caracterizada por taquipnea, de curso corto y autolimitado); hiperbilirrubinemia indirecta (incremento de bilirrubinemia sobre $0,5 \mathrm{mg} / \mathrm{dl}$ por hora durante el primer día de vida, o valores de bilirrubinemia que requiera fototerapia); sospecha de sepsis (paciente con factores de riesgo para infección: rotura de membranas $>18$ horas, fiebre materna $>38^{\circ} \mathrm{C}$ 
durante parto, corioamnionitis; madre con infección urinaria no tratada o historia de hijos con sepsis por Streptococo grupo B; niño con signos clínicos: fiebre, hipoxemia, hipotermia, bradicardia, palidez o hipotonía y/o laboratorio con proteína $\mathrm{C}$ reactiva $>1 \mathrm{mg} / \mathrm{dL}$ o neutrofilia absoluta o trombocitopenia $<80.000 / \mathrm{mm} 3$ ); sepsis confirmada (presencia de síndrome de respuesta inflamatoria sistémica, con hemocultivos positivos); neumonía (dificultad respiratoria asociada a radiografía de tórax compatible); depresión respiratoria (Test de Apgar $<6$ a los 5 minutos y/o necesidad de ventilación con presión positiva > 2 minutos y/o necesidad de intubación en atención inmediata); hipotermia (temperatura axilar bajo $36.5^{\circ} \mathrm{C}$.); hipoglicemia (glicemia $<45 \mathrm{mg} / \mathrm{dL}$ en primeras 24 horas y $<$ de $50 \mathrm{mg} / \mathrm{dL}$ después); dificultad en la alimentación (discordinación entre succión/deglución/respiración que dificulta la alimentación del recién nacido); enterocolitis necrotizante (distensión abdominal y sangrado rectal junto con radiografía que muestra neumatosis intestinal); cianosis; y, apnea (interrupción del flujo aéreo por al menos 20 segundos o menos si se acompaña de bradicardia y/o cianosis).

Para el análisis estadístico comparamos la frecuencia de los resultados perinatales entre los PT (34 0/7 - 36 6/7 semanas) y los nacidos a término completo (39 0/7 - 40 6/7 semanas), utilizando la prueba de Chi-cuadrado y calculando Odds Ratio (OR). Se considera significación estadística un valor $\mathrm{p}<0,05$.

\section{RESULTADOS}

Entre enero 2009 y diciembre 2012, nacieron 8.890 niños vivos, con una tasa de partos prematuros de $11,42 \%(n=1.015)$, de los cuales $65,62 \%$ corresponden a partos PT $(n=666)$; la tasa de parto PT fue de $7,49 \%$. Entre los PT, un $16,37 \%$ correspondieron a niños nacidos a las 34 semanas de edad gestacional (34 0/7 - $346 / 7$ ), 27,78\% a las 35 y $55,85 \%$ a las 36 semanas. Los recién nacidos de término completo (39 0/7 y 40 6/7), fueron 4.091, lo que equivale a un $46,02 \%$ de todos los nacidos vivos en nuestra institución (Tabla I).
Entre los recién nacidos (excluidos los que presentan malformaciones congénitas graves, aneuploidías y hospitalizados por causa materna), fueron hospitalizados durante el primer mes de vida el $50,15 \%$ de niños PT $(n=342)$, y el $10,10 \%$ de los niños de término completo $(n=413)(p<0,05)$. En la Tabla II se muestra el grupo clínico al que pertenecen los niños PT que fueron hospitalizados, destacando que el 35,89\% fueron por indicación médica. Observamos que en el grupo indicación médica, un $19,25 \%$ (46 niños), no tenían una indicación basada en la evidencia. Entre las patologías médicas que motivaron la interrupción del embarazo antes de lo recomendado destaca: el oligoamnios aislado antes de las 36 semanas; los síndromes hipertensivos del embarazo que no corresponden a preeclampsia; embarazo gemelar bicorial biamniótico sin complicaciones y el antecedente de óbito fetal. No encontramos ninguna interrupción del embarazo puramente electiva.

En las Tablas III y IV se observan los resultados perinatales de los niños PT y se comparan con los nacidos a término completo. El grupo de PT tiene casi 9 veces más riesgo de ser hospitalizado, llegando a ser de 48 veces en el caso de los niños de 34 semanas y 12 veces a las 35 semanas. Los PT presentan significativamente mayor morbilidad perinatal en casi todas las patologías estudiadas, siendo el riesgo mayor a menor edad gestacional.

\section{DISCUSIÓN}

En nuestro Hospital, entre los años 2009 y 2012, los PT correspondieron al 7,49 \% de todos los recién nacidos vivos. Al analizar el grupo clínico de parto prematuro, un $64,11 \%$ fue debida a un parto prematuro idiopático o asociado a rotura de membranas y un $35,89 \%$ correspondió al grupo de indicación médica. Dentro del grupo de indicaciones médicas detectamos que en un $19,25 \%$ de los casos la decisión fue no basada en la evidencia; no detectamos casos de interrupción puramente electiva. Los PT, comparados con los niños de término completo, tienen significativamente más riesgo de hospitalización y morbilidad neonatal, siendo este riesgo mayor a menor edad gestacional.

Tabla I

NACIDOS VIVOS HOSPITAL CLÍNICO PONTIFICIA UNIVERSIDAD CATÓLICA DE CHILE, 2009 - 2012

\begin{tabular}{lccccc}
\hline & 2009 & 2010 & 2011 & 2012 & Total \\
\hline Total nacidos vivos n & 2.342 & 2.247 & 2.206 & 2.095 & 8.890 \\
Partos prematuros n (\%) & $259(11,06)$ & $259(11,53)$ & $257(11,65)$ & $240(11,46)$ & $1.015(11,42)$ \\
Partos prematuros tardíos n (\%) & $191(8,16)$ & $152(6,9)$ & $167(7,57)$ & $156(7,45)$ & $666(7,49)$ \\
\hline
\end{tabular}


Tabla II

GRUPOS CLÍNICOS DE PREMATUREZ DE LOS PREMATUROS TARDÍOS

\begin{tabular}{lcc}
\hline & $\mathrm{n}$ & $\%$ \\
\hline Total de partos prematuros (PP) & 666 & 100 \\
PP idiopáticos & 276 & 41,44 \\
PP asociados a RPM & 151 & 22,67 \\
PP por indicación médica & 239 & 35,89 \\
Basado en la evidencia & 193 & 80,75 \\
No basado en la evidencia & 46 & 19,25 \\
Puramente electivo & 0 & 0
\end{tabular}

PP: parto prematuro. RPM: rotura prematura de membranas.

\section{Tabla III}

COMPARACIÓN ENTRE LOS PREMATUROS TARDÍOS Y LOS NIÑOS DE TÉRMINO COMPLETO

\begin{tabular}{|c|c|c|}
\hline & $\begin{array}{c}\text { Nacidos entre las } 34 \text { a } 36 \\
\text { semanas }(n=666) \\
\% \\
\text { OR }(\text { IC } 95 \%)\end{array}$ & $\begin{array}{c}\text { Nacidos entre las } 39 \text { a } 40 \\
\text { semanas }(n=4.091) \\
\%\end{array}$ \\
\hline Necesidad de hospitalización & $\begin{array}{c}50,15 \\
8,96(7,46-10,76)\end{array}$ & 10,10 \\
\hline Hospitalización para observación & $\begin{array}{c}2,85 \\
11,98(5,55-25,89)\end{array}$ & 0,24 \\
\hline Síndrome de distrés respiratorio & $\begin{array}{c}2,25 \\
11,76(4,97-27,85)\end{array}$ & 0,20 \\
\hline Enfermedad de membrana hialina & $\begin{array}{c}2,85 \\
246,44(14,86-4.086,74)\end{array}$ & 0 \\
\hline Taquipnea transitoria neonatal & $\begin{array}{c}4,35 \\
18,58(9,01-38,31)\end{array}$ & 0,24 \\
\hline Neumotórax & $\begin{array}{c}0,3 \\
3,08(0,56-16,84)\end{array}$ & 0,10 \\
\hline Resultado respiratorio compuesto ${ }^{a}$ & $\begin{array}{c}9,76 \\
20(12,24-32,68)\end{array}$ & 0,54 \\
\hline Hiperbilirrubinemia indirecta & $\begin{array}{c}32,58 \\
9,30(7,50-11,54)\end{array}$ & 4,94 \\
\hline Sepsis confirmada & $\begin{array}{c}1,05 \\
3,94(1,52-10,20)\end{array}$ & 0,27 \\
\hline Sospecha de sepsis & $\begin{array}{c}7,81 \\
5,89(4,01-8,64)\end{array}$ & 1,42 \\
\hline Neumonia & $\begin{array}{c}1,05 \\
10,85(3,17-37,18)\end{array}$ & 0,10 \\
\hline Depresión respiratoria & $\begin{array}{c}2,7 \\
4,34(2,37-7,97)\end{array}$ & 0,64 \\
\hline Hipotermia & $\begin{array}{c}4,65 \\
15,31(7,97-29,42)\end{array}$ & 0,32 \\
\hline Hipoglicemia & $\begin{array}{c}3,15 \\
2,58(1,54-4,32)\end{array}$ & 0,37 \\
\hline
\end{tabular}


Continuación Tabla III

\begin{tabular}{lcc}
\hline & $\begin{array}{c}\text { Nacidos entre las } 34 \text { a } 36 \\
\text { semanas }(\mathrm{n}=666) \\
\% \\
\text { OR }(\mathrm{IC} 95 \%)\end{array}$ & $\begin{array}{c}\text { Nacidos entre las 39 a } 40 \\
\text { semanas }(\mathrm{n}=4.091) \\
\%\end{array}$ \\
\hline Dificultad alimentación & 5,26 & 0,68 \\
Enterocolitis necrotizante & $8,05(4,86-13,32)$ & 0,07 \\
Cianosis & 0,9 & 0,22 \\
Apnea & $1,39(3,09-49,65)$ & 0,15 \\
Asfixia & $4,82(1,79-12,98)$ & 0,12 \\
Convulsiones & 5,41 & $0,90(16,33-92,71)$ \\
& 0,3 & 0,22 \\
\hline
\end{tabular}

a. Respiratorio compuesto incluye: síndrome de distrés respiratorio, enfermedad de membrana hialina, taquipnea transitoria neonatal y neumotórax.

\section{Tabla IV}

\section{COMPARACIÓN ENTRE LOS PREMATUROS TARDÍOS Y LOS NIÑOS DE TÉRMINO COMPLETO, SEGÚN EDAD GESTACIONAL}

\begin{tabular}{|c|c|c|c|c|}
\hline & $\begin{array}{c}34 \text { semanas }(n=109) \\
\% \\
\text { OR }(I C 95 \%)\end{array}$ & $\begin{array}{c}35 \text { semanas }(n=185) \\
\% \\
\text { OR }(I C 95 \%)\end{array}$ & $\begin{array}{c}36 \text { semanas }(n=372) \\
\% \\
\text { OR }(I C 95 \%)\end{array}$ & $\begin{array}{c}39 \text { a } 40 \text { semanas } \\
(\mathrm{n}=4.091) \\
\%\end{array}$ \\
\hline $\begin{array}{l}\text { Necesidad de } \\
\text { hospitalización }\end{array}$ & $\begin{array}{c}84,4 \\
48,19(28,44-81,66)\end{array}$ & $\begin{array}{c}58,38 \\
12,49(9,17-17,02)\end{array}$ & $\begin{array}{c}36,02 \\
5,01(3,96-6,34)\end{array}$ & 10,1 \\
\hline $\begin{array}{l}\text { Hospitali- } \\
\text { zación para } \\
\text { observación }\end{array}$ & $\begin{array}{c}6,42 \\
28,01(10,45-75,05)\end{array}$ & $\begin{array}{c}4,32 \\
18,45(7,19-47,30)\end{array}$ & $\begin{array}{c}1,08 \\
4,44(1,38-14,21)\end{array}$ & 0,24 \\
\hline $\begin{array}{l}\text { Síndrome de } \\
\text { distrés } \\
\text { respiratorio }\end{array}$ & $\begin{array}{c}4,59 \\
24,54(7,89-76,28)\end{array}$ & $\begin{array}{c}3,78 \\
20,07(7,20-55,96)\end{array}$ & $\begin{array}{c}0,81 \\
4,15(1,10-15,71)\end{array}$ & 0,2 \\
\hline $\begin{array}{l}\text { Enfermedad } \\
\text { de membrana } \\
\text { hialina }\end{array}$ & $\begin{array}{c}8,26 \\
773,52(44,71-13.382,36)\end{array}$ & $\begin{array}{c}3,24 \\
26,32(16,63-5.280,71)\end{array}$ & $\begin{array}{c}1,08 \\
99,9(5,36-1.859,7)\end{array}$ & 0 \\
\hline $\begin{array}{l}\text { Taquipnea } \\
\text { transitoria } \\
\text { neonatal }\end{array}$ & $\begin{array}{c}11,01 \\
50,49(21,30-119,67)\end{array}$ & $\begin{array}{c}4,32 \\
18,45(7,19-47,30)\end{array}$ & $\begin{array}{c}2,42 \\
10,12(4,09-25,06)\end{array}$ & 0,24 \\
\hline Neumotórax & $\begin{array}{c}1,83 \\
19,10(3,46-105,41)\end{array}$ & $\begin{array}{c}0 \\
2,44(0,13-45,65)\end{array}$ & $\begin{array}{c}0 \\
1,21(0,66-22,69)\end{array}$ & 0,1 \\
\hline $\begin{array}{l}\text { Resultado } \\
\text { respiratorio } \\
\text { compuesto }^{\text {a }}\end{array}$ & $\begin{array}{c}25,69 \\
63,93(35,08-116,52)\end{array}$ & $\begin{array}{c}11,35 \\
23,68(12,77-43,94)\end{array}$ & $\begin{array}{c}4,3 \\
8,31(4,33-15,97)\end{array}$ & 0,54 \\
\hline $\begin{array}{l}\text { Hiperbili- } \\
\text { rrubinemia } \\
\text { indirecta }\end{array}$ & $\begin{array}{c}50,46 \\
19,61(13,13-29,29)\end{array}$ & $\begin{array}{c}41,08 \\
13,42(9,70-18,58)\end{array}$ & $\begin{array}{c}23,12 \\
5,79(4,38-7,66)\end{array}$ & 4,94 \\
\hline
\end{tabular}


Continuación Tabla IV

\begin{tabular}{|c|c|c|c|c|}
\hline & $\begin{array}{c}34 \text { semanas }(n=109) \\
\% \\
\text { OR }(\text { IC } 95 \%)\end{array}$ & $\begin{array}{c}35 \text { semanas }(n=185) \\
\% \\
\text { OR }(I C 95 \%)\end{array}$ & $\begin{array}{c}36 \text { semanas }(n=372) \\
\% \\
\text { OR }(\text { IC } 95 \%)\end{array}$ & $\begin{array}{c}39 \text { a } 40 \text { semanas } \\
(n=4.091) \\
\%\end{array}$ \\
\hline $\begin{array}{l}\text { Sepsis } \\
\text { confirmada }\end{array}$ & $\begin{array}{c}0,92 \\
3,43(0,44-26,84)\end{array}$ & $\begin{array}{c}1,08 \\
4,05(0,89-18,42)\end{array}$ & $\begin{array}{c}1,08 \\
4,03(1,28-12,72)\end{array}$ & 0,27 \\
\hline $\begin{array}{l}\text { Sospecha de } \\
\text { sepsis }\end{array}$ & $\begin{array}{c}22,02 \\
19,63(11,65-33,09)\end{array}$ & $\begin{array}{c}9,19 \\
7,04(4,01-12,34)\end{array}$ & $\begin{array}{c}2,96 \\
2,12(1,10-4,07)\end{array}$ & 1,42 \\
\hline Neumonia & $\begin{array}{c}1,83 \\
19,10(3,46-105,41)\end{array}$ & $\begin{array}{c}1,62 \\
16,84(3,74-75,81)\end{array}$ & $\begin{array}{c}0,54 \\
5,52(1,01-30,25)\end{array}$ & 0,1 \\
\hline $\begin{array}{l}\text { Depresión } \\
\text { respiratoria }\end{array}$ & $\begin{array}{c}5,5 \\
9,11(3,67-22,60)\end{array}$ & $\begin{array}{c}3,78 \\
6,15(2,63-14,63)\end{array}$ & $\begin{array}{c}1,34 \\
2,13(0,81-5,58)\end{array}$ & 0,64 \\
\hline Hipotermia & $\begin{array}{c}6,42 \\
24,53(8,41-55,09)\end{array}$ & $\begin{array}{c}6,49 \\
21,76(9,78-48,39)\end{array}$ & $\begin{array}{c}3,23 \\
10,46(4,74-23,09)\end{array}$ & 0,32 \\
\hline Hipoglicemia & $\begin{array}{c}5,5 \\
4,61(1,94-11,00)\end{array}$ & $\begin{array}{c}2,7 \\
2,20(0,87-5,58)\end{array}$ & $\begin{array}{c}2,69 \\
2,19(1,10-4,35)\end{array}$ & 0,37 \\
\hline $\begin{array}{l}\text { Dificultad } \\
\text { alimentación }\end{array}$ & $\begin{array}{c}6,42 \\
9.96(4.25-23.33)\end{array}$ & $\begin{array}{c}6,49 \\
10,07(5,03-20,13)\end{array}$ & $\begin{array}{c}4,3 \\
6,52(3,50-12,17)\end{array}$ & 0,68 \\
\hline $\begin{array}{l}\text { Enterocolitis } \\
\text { necrotizante }\end{array}$ & $\begin{array}{c}3,67 \\
51,91(11,47-234,86)\end{array}$ & $\begin{array}{c}0,54 \\
7,41(0,77-71,54)\end{array}$ & $\begin{array}{c}0,27 \\
3,67(0,38-35,40)\end{array}$ & 0,07 \\
\hline Cianosis & $\begin{array}{c}0 \\
1,96(0,11-33,93)\end{array}$ & $\begin{array}{c}0,54 \\
2,46(0,31-19,56)\end{array}$ & $\begin{array}{c}1,61 \\
7,44(2,63-21,01)\end{array}$ & 0,22 \\
\hline Apnea & $\begin{array}{c}19,27 \\
162,47(64-412,43)\end{array}$ & $\begin{array}{c}4,86 \\
34,82(12,26-98,89)\end{array}$ & $\begin{array}{c}1,61 \\
11,16(3,58-34,78)\end{array}$ & 0,15 \\
\hline Asfixia & $\begin{array}{c}0,92 \\
7,57(0,88-65,32)\end{array}$ & $\begin{array}{c}0,54 \\
4,44(0,52-38,21)\end{array}$ & $\begin{array}{c}0 \\
0,99(0,06-18,07)\end{array}$ & 0,12 \\
\hline Convulsiones & $\begin{array}{c}0 \\
1,96(0,11-33,93)\end{array}$ & $\begin{array}{c}0,54 \\
2,46(0,31-19,56)\end{array}$ & $\begin{array}{c}0,27 \\
1,22(0,15-9,68)\end{array}$ & 0,22 \\
\hline
\end{tabular}

a. Respiratorio compuesto incluye: síndrome de distrés respiratorio, enfermedad de membrana hialina, taquipnea transitoria neonatal y neumotórax.

Nuestras tasas de nacimientos prematuros $(11,42 \%)$ y PT $(7,49 \%)$, son similares a las publicadas en la literatura (17), sin embargo son mucho mayores a la media nacional (1). Postulamos que la mayor tasa de partos prematuros en nuestra entidad se debe a que contamos con una unidad de medicina materno-fetal y neonatología de alta complejidad y con un equipo especializado, al que derivan pacientes con embarazos de alto riesgo.

Cuando analizamos cada año del estudio (ver Tabla I), no encontramos un cambio significativo en las tasas de prematuridad en ningún grupo de prematuros, a diferencia de lo publicado en el extranjero en que en años recientes la tasa de partos prematuros va en aumento (9-11). Pensamos que la ausencia de cambio en nuestro centro puede explicarse por el corto período observado, o bien porque nuestro hospital corresponde a un centro universitario, con auditoría interna sobre las indicaciones de interrupción del embarazo y apego a las normas clínicas basadas en la mejor evidencia disponible. 
Las sociedades americanas de obstetricia y pediatría han invitado a analizar las causas de interrupciones por causa médica del embarazo en el grupo de PT, porque se ha encontrado que hasta $23 \%$ de éstas, no tiene un registro acerca de la causa de la interrupción (18). Laugon y cols (13) han demostrado que $18 \%$ de las interrupciones por causa médica del embarazo en los PT tenía una indicación débil y un $1 \%$ eran puramente electivos, mientras que Holland y cols (12), identificaron en sus estadísticas que hasta un $10 \%$ eran puramente electivas. Gyamfi-Bannerman y cols (14) encontraron que el $56,7 \%$ de las interrupciones por indicación médica del embarazo, no estaban avaladas por la evidencia.

Al estudiar las causas de interrupción de los PT en nuestro centro, observamos que un $64 \%$ corresponden al inicio espontáneo del trabajo de parto o están asociados a la rotura prematura de membranas, por lo que no es posible evitar el parto prematuro en éstas circunstancias. Si bien existen algunos grupos que están evaluando un manejo expectante en la rotura de membranas después de las 34 semanas, o el uso de tocolíticos y corticoides en este grupo de niños -con el objeto de disminuir el número de partos PT o sus complicacioneshasta el momento, ninguna de estas estrategias ha mostrado un beneficio (19-27).

Cuando evaluamos las indicaciones médicas de interrupción del embarazo en nuestra serie, intentando encontrar causas prevenibles, observamos que un $19,25 \%$ no estaban basadas en la evidencia y no hubo ninguna exclusivamente electiva. Las indicaciones no basadas en la evidencia parecen estar soportadas en la experiencia clínica más que por criterios científicos. Si bien estos datos son alentadores, pues las indicaciones médicas no basadas en la evidencia están en el límite inferior de lo reportado internacionalmente, nos muestran que en el grupo de niños PT, 1 de cada 5 interrupciones electivas del embarazo pudo evitarse y llegar a una edad gestacional mayor. Pensamos que este número de indicaciones médicas no basadas en la evidencia puede deberse a presión familiar, profesional y legal, lo que podría llevar a tomar medidas excedidas con el objeto de obtener un recién nacido vivo, sin considerar los riesgos a corto y largo plazo de esos niños, que si bien son bajos en términos absolutos, son mucho más altos que en los niños de término.

Hoy día, de acuerdo a toda la evidencia disponible, no existen dudas acerca de que los niños PT tienen más necesidades de hospitalización y más alta frecuencia de morbilidad perinatal, como lo reafirma nuestro estudio y otro publicado en nuestro país (15); a menor edad gestacional son mayores las complicaciones neonatales. Todos los resultados perinatales estudiados en el grupo de PT fueron mayores que en el grupo de niños na- cidos a término, sin embargo no encontramos que esas diferencias fueran significativas en las tasas de neumotórax, convulsiones y asfixia. A nuestro juicio, esta falta de significación estadística podría deberse al número de pacientes estudiados y a la baja frecuencia de estas patologías.

Llama la atención en nuestra serie que las tasas de hipotermia, dificultad para la alimentación y cianosis, la frecuencia de ellas era mayor en niños nacidos a las 35 que a las 34 semanas de edad gestacional; pensamos que esto último se podría deber a que son significativamente más los niños de 34 semanas que quedan hospitalizados inmediatamente $\mathrm{y}$, como presentan patologías más serias, no se consignan estos problemas en la epicrisis neonatal o, que no los desarrollan por estar bajo el cuidado neonatal, a diferencia de los niños de 35 semanas, que presentan menor frecuencia de patologías serias, por lo que quedan menos hospitalizados y presentan estas patologías luego del alta.

\section{CONCLUSIONES}

Nuestro estudio confirma que los niños PT tienen más morbilidad perinatal y mayores necesidades de hospitalización que los niños de término completo, recordando la importancia que tiene el prevenir este tipo de partos prematuros. En el Hospital Clínico de la Universidad Católica de Chile, las interrupciones del embarazo no basadas en la evidencia o puramente electivas, si bien están en el límite inferior de lo reportado internacionalmente, nos imponen como desafío reducirlas o hacerlas desaparecer, pues constituyen un grupo de partos prematuros potencialmente evitables. Si bien un importante grupo de partos PT no se pueden evitar (indicaciones médicas correctamente avaladas en evidencia sólida), es necesario que cada centro analice periódicamente las causas de interrupción médica de sus PT, con el objeto de realizar medidas tendientes a reducir las interrupciones no basadas en la evidencia o puramente electivas, si es que las hubiere; esta simple actividad pudiera reducir el riesgo de morbilidad y mortalidad de este grupo de recién nacidos.

\section{REFERENCIAS}

1. González R, Nien JK, Vera C, Poblete JA, Carvajal J, González M, et al. ¿Existe un aumento de los nacimientos en Chile en el período 2000-2009?: Análisis de los principales indicadores materno-infantiles de la década. Rev Chil Obstet Ginecol. 2011;76(6):404-11.

2. Teune MJ, Bakhuizen S, Gyamfi Bannerman C, Opmeer B, van Kaam A, van Wassenaer A, et al. A systematic review of severe morbidity in infants born late preterm. Am J Obstet Gynecol. 2011;205(4)374.e1-9.

3. Wang ML, Dorer DJ, Fleming MP, Catlin EA. Clinical outcomes of near-term infants. Pediatrics. 2004;114(2):372-6. 
4. McLaurin KK, Hall CB, Jackson EA, Owens OV, Mahadevia PJ. Persistence of morbidity and cost differences between late-preterm and term infants during the first year of life. Pediatrics. 2009;123(2):653-9.

5. Underwood MA, Danielsen B, Gilbert WM. Cost, causes and rates of rehospitalization of preterm infants. J Perinatol. 2007;27(10):614-9.

6. Petrini JR, Dias T, McCormick MC, Massolo ML, Green NS, Escobar GJ. Increased risk of adverse neurological development for late preterm infants. J Pediatr. 2009;154(2):169-76.

7. Chyi LJ, Lee HC, Hintz SR, Gould JB, Sutcliffe TL. School outcomes of late preterm infants: special needs and challenges for infants born at 32 to 36 weeks gestation. J Pediatr. 2008;153(1):25-31.

8. Moster, D, Lie RT, Markestad, T. Long-term medical and social consequences of preterm birth. $\mathrm{N}$ Engl $\mathrm{J}$ Med. 2008;359(3):262-73.

9. Davidoff MJ, Dias T, Damus K, Russell R, Bettegowda VR, Dolan S, et al. Changes in the gestational age distribution among U.S. singleton births: impact on rates of late preterm birth, 1992 to 2002. Semin Perinatol. 2006;30(1):8-15.

10. Martin JA, Hamilton BE, Ventura SJ, Osterman MJ, Kirmeyer S, Mathews TJ, et al. Births: final data for 2009. Natl Vital Stat Rep. 2011;60(1):1-70.

11. Ananth, CV, Joseph, KS, Oyelese, Y, Demissie K, Vintzileos AM. Trends in Preterm Birth and Perinatal Mortality Among Singletons: United States, 1989 Through 2000. Obstet Gynecol. 2005;105(5 Pt 1):1084-91.

12. Holland MG, Refuerzo JS, Ramin SM, Saade GR, Blackwell SC. Late preterm birth: how often is it avoidable? Am J Obstet Gynecol. 2009;201(4):404.e1-4.

13. Laughon SK, Reddy UM, Sun L, Zhang J. Precursors for late preterm birth in singleton gestations. Obstet Gynecol. 2010;116(5):1047-55.

14. Gyamfi-Bannerman C, Fuchs KM, Young OM, Hoffman MK. Nonspontaneous late preterm birth: etiology and outcomes. Am J Obstet Gynecol. 2011;205(5):456.e1-6.

15. Valdés E, Sepúlveda A, Catalán J, Reyes A. Morbilidad neonatal de los prematuros tardíos en embarazos únicos: estudio de caso-control. Rev Chil Obstet Ginecol. 2012;77(3):195-200.

16. Spong C, Mercer B, D'Alton M, Kilpatrick S, Blackwell S, Saade G. Timing of indicated late-preterm and early-term birth. Obstet Gynecol. 2011;118(2 Pt 1):323-33.

17. Goldenberg, RL, Culhane, JF, lams, JD, Romero, R. Epidemiology and causes of preterm birth. Lancet. 2008;371(9606):75-84.

18. Reddy U, Ko CW, Raju TN, Willinger M. Delivery Indications at Late-Preterm Gestations and Infant Mortality Rates in the United States Pediatrics. 2009;124(1):234-40.

19. Gyamfi-Bannerman C. Late preterm birth: management dilemmas. Obstet Gynecol Clin N Am. 2012;39(1):35-45.

20. lams J, Donovan E. Spontaneous late preterm births: what can be done to improve outcomes. Semin Perinatol. 2011;35(5):309-13.

21. Morris JM, Roberts CL, Crowther CA, Buchanan SL, Henderson-Smart DJ, Salkeld G. Protocol for the immediate delivery versus expectant care of women with preterm prelabour rupture of the membranes close to term (PPROMT) Trial [ISRCTN44485060]. BMC Pregnancy Childbirth. 2006;6:9.

22. van der Ham DP, van der Heyden JL, Opmeer BC, Mulder AL, Moonen RM, van Beek JH, et al. Management of late-preterm premature rupture of membranes: the PPROMEXIL-2 trial. Am J Obstet Gynecol. 2012;207(4):276.e1-10.

23. Porto AM, Coutinho IC, Correia JB, Amorim MM. Effectiveness of antenatal corticosteroids in reducing respiratory disorders in late preterm infants: randomised clinical trial. BMJ. 2011;342:d1696.

24. Kamath-Rayne B, DeFranco E, Marcotte M. Antenatal steroids for treatment of fetal lung immaturity after 34 weeks of gestation an evaluation of neonatal outcomes. Obstet Gynecol. 2012;119(5):909-16.

25. Gyamfi-Bannerman C, Gilbert S, Landon M, Spong C, Rouse D, Varner M, et al. Effect of antenatal corticosteroids on respiratory morbidity in singletons after latepreterm birth. Obstet Gynecol. 2012;119(3):555-9.

26. Singh N, Singh U, Seth S. Comparative study of nifedipine and isoxpurine as tocolytics for preterm labor. $\mathrm{J}$ Obstet Gynaecol India. 2011;61(5):512-5.

27. Hehir MP, O'Connor HD, Kent EM, Robson MS, Keane DP, Geary MP, et al. Early and late preterm delivery rates - a comparison of differing tocolytic policies in a single urban population. J Matern Fetal Neonatal Med. 2012;25(11):2234-6. 